\title{
Light splitting in nanoporous gold and silver
}

\author{
Michel Bosman $^{1 *}$, Geoffrey R. Anstis ${ }^{2}$, Vicki J. Keast ${ }^{3}$, Jackson D. Clarke, ${ }^{3}$ and Michael B. Cortie ${ }^{2}$ \\ ${ }^{1}$ Institute of Materials Research and Engineering, A*STAR (Agency for Science, Technology and \\ Research), 3 Research Link, 117602, Singapore \\ ${ }^{2}$ Institute for Nanoscale Technology, University of Technology, Sydney, NSW 2007, Australia \\ ${ }^{3}$ School of Mathematical and Physical Sciences, The University of Newcastle, Callaghan NSW 2308, \\ Australia \\ *Address correspondence to: michel.bosman@gmail.com
}

\begin{abstract}
Nanoporous gold and silver exhibit strong, omnidirectional broadband absorption in the far-field. Even though they consist entirely of gold or silver atoms, these materials appear black and dull, in great contrast with the familiar luster of continuous gold and silver. The nature of these anomalous optical characteristics is revealed here by combining nanoscale electron energy-loss spectroscopy with direct dipole- and boundary element simulations. It is established that the strong broadband absorption finds its origin in nanoscale splitting of light; with great local variations in the absorbed color. This nanoscale polychromaticity results from the excitation of localized surface plasmon resonances, which are imaged and analyzed here with deep sub-wavelength, nanometer spatial resolution. We demonstrate that with this insight, it is possible to customize the absorbance and reflectance wavelength bands of thin nanoporous films by only tuning their morphology.
\end{abstract}

KEYWORDS: Plasmonics, Optical properties of nanostructures, electron energy-loss spectroscopy, Scanning transmission electron microscopy 
Nanoporous gold or silver, also called 'black gold' or 'black silver' consist entirely of gold or silver atoms, but due to their highly porous and corrugated morphology, are black in appearance, and have a dull instead of shiny luster. They are extreme examples of metals with optical properties that are highly modified solely due to altered surface morphologies. Such irregular structures are increasingly wellunderstood theoretically, ${ }^{1-3}$ but have so far proven difficult to investigate experimentally at subwavelength dimensions. In this work, it will be shown that in nanoporous gold and silver, the far-field, macroscopic broadband absorption characteristics result from local variations in the color of the absorbed light. Plane-wave simulations and electron-beam spectroscopy will be used to demonstrate that localized surface plasmon resonances are responsible for this nanoscale polychromaticity.

Surface plasmons are collectively oscillating conduction electrons at a metal surface. Their hybrid nature combines photonic wave properties with electron kinetics, giving them potentially widespread application in bridging the disparity in length scale between photonics and electronics. ${ }^{4}$ Nonpropagating surface plasmon resonances are known as localized surface plasmons, and can be used to concentrate light and change the optical properties of a material at deep sub-wavelength dimensions. ${ }^{5,6}$ In order to provide the missing momentum that is needed to excite surface plasmons with light, a periodic array of surface features ${ }^{7}$ or small non-periodic structures such as protruding bumps or particles can be used. Depending on the shape, size, and dielectric properties of the metal and its environment, the plasmons will oscillate at a specific dipole resonance frequency.

The local electric field enhancements associated with surface plasmons are most commonly imaged with dark field microscopy ${ }^{8}$ and near-field scanning optical microscopy (NSOM). ${ }^{9,10}$ However, the spatial resolution of these optical techniques is typically $10-50 \mathrm{~nm}$, often too coarse to observe the rich details and local distribution of plasmon modes. (Scanning) transmission electron microscopy, (S)TEM, on the other hand, can provide nanometer spatial resolution, as well as the energy and momentum needed for plasmon excitation. The direct electrostatic interaction of a fast electron with the surface electrons allows a general excitation scheme: elastic and inelastic scattering of the fast electrons can transfer nearly any amount of energy and momentum to a metal surface. In fact, the first experimental observations of (what were later called) surface plasmons were made with electron microscopes, by detecting the energy that is lost from the primary electron beam when it transmits through thin metal foils, using electron energy-loss spectroscopy (EELS). ${ }^{11}$ These losses were later theoretically described to arise from collective plasma oscillations ${ }^{12}$ resulting from the existence of non-vanishing electric fields at metal surfaces. ${ }^{13}$ Early demonstrations of spatially-resolved EELS ${ }^{14,15}$ confirmed the localized nature of surface plasmons on metal particles. Thanks to the steady instrumental improvements in electron optics and fast, parallel EELS acquisition, it has recently been demonstrated that surface plasmon modes 
can be mapped with nanometer spatial resolution and spectral sensitivity down into the visible $e^{16,17}$ and even infrared. ${ }^{18,19}$

In this Article, nanoporous gold and silver will be investigated with STEM-EELS, using a new imaging scheme that combines plasmon maps with STEM dark-field images. Boundary-element and direct-dipole simulations will be used to explain some unexpected experimental observations, and will also relate the EELS maps with plasmon modes that are excited by far-field, plane-wave illumination.

\section{RESULTS AND DISCUSSION}

Plasmon mapping with STEM EELS. Figure 1 presents a scheme for imaging variations in absorption at deep sub-wavelength dimensions. The combination of EELS plasmon maps with highangle, annular dark field (HAADF) STEM images of the same area preserves in one image the correlation between surface plasmon modes and the local geometry of the metal.

A $77 \times 97 \mathrm{~nm}$ area from a thin nanoporous silver film is shown. In STEM imaging, a fast beam of electrons travels close to or through the specimen material; for heavy elements such as gold and silver, a large number of these electrons will scatter elastically to relatively high angles, giving rise to a strong HAADF signal, as in Fig. 1a. Some fast electrons will scatter inelastically instead; they can for example slow down due to the excitation of surface plasmons. If the local conditions are right for the excitation of such a surface plasmon, a distinct peak will be observed in the EELS spectrum, at the plasmon resonance energy. The plasmon excitation probability can be spatially mapped by acquiring EELS spectra in a rectangular raster of pixels, and selecting in each pixel a narrow energy window around the plasmon energy. Fig. 1b-1d were obtained in this manner for the three surface plasmons that were resonant in this small area. Each EELS plasmon map can be colour-coded according to the colour of light that would be absorbed at that energy, in this case orange for the mode at $1.90 \mathrm{eV}$, blue at $2.90 \mathrm{eV}$, and violet for the near-ultraviolet absorption at $3.45 \mathrm{eV}$. When the STEM HAADF image and EELS plasmon maps are overlaid, as in Fig. 1e, morphological information is directly visually associated with the energy and spatial distribution of the localized surface plasmons. This concise imaging scheme will be used in several figures in this work. One point to note in Figure 1 is the orange-coded plasmon mode at $1.90 \mathrm{eV}$. With its single maximum at the centre of the thin silver bridge, it is likely the result of a tripolar plasmon mode, with charge oscillating antiparallelly between the anti-node at the centre and the two nodes at the bases of the silver bridge. A tripolar plasmon mode at these length scales necessarily has to be a 'dark' plasmon mode ${ }^{20}$ as it is incompatible with the uniformity of the electric field of light at these length scales, given that the half-wavelength of light is $326 \mathrm{~nm}$ at this energy. Dark plasmon modes are only accessible with near-field techniques such as NSOM or EELS, ${ }^{21}$ or under specific geometrical circumstances. ${ }^{22}$ 
Covering a much larger area of $720 \times 500 \mathrm{~nm}$, Fig. 2a shows an example of the rich variety in plasmon modes that can occur in porous or irregularly shaped silver. For this area, five main plasmon oscillation modes were observed, but several much weaker modes are also present. Fig. 2b-c show the abundance of plasmonic activity in more detail, by displaying the variation in EELS spectra over a 300 $\mathrm{nm}$ long trace along the surface. At many locations, more than one plasmon mode is present, shown as multiple peaks in the EELS spectra. Despite this seeming complexity, general trends can be observed. Most obviously, well-defined geometrical structures in the silver such as protrusions, convex features and elongations have distinct plasmon modes. Low-energy modes at near-infrared frequencies are associated with large features and they extend over large distances in space in comparison with highenergy modes; this delocalization effect ${ }^{23}$ appeared in earlier published work as well. ${ }^{24,25}$ In Fig. $2 \mathrm{c}$, delocalization becomes apparent in the abrupt change of the high-energy modes over short distances, while the low-energy modes change more gradually over longer distances. Only the dominant plasmon mode at a certain location usually appears in the plasmon maps. However, at several locations, two or more modes can have similar amplitudes, giving rise to mixed colors such as orange and white in the maps. Possible dark modes at elongated silver structures are indicated with asterisks.

Far-field optical vs. near-field EELS. At first glance, the multitude of local plasmon modes in irregular and nanoporous metal surfaces appears to be analogous to plasmons in small metal particles. Both for far-field and near field responses, it is well-known that by varying the size and shape of small metal particles, their resonant plasmon modes can be tuned. ${ }^{26}$ Fig. 3a shows an example of this effect for gold and silver oblate spheroids; an increasing width/height aspect ratio lowers the dominant (axial) plasmon frequency. Besides single-particle modes, it has been shown that plasmon coupling between adjacent metal volumes can also result in strongly modified EELS and optical spectra. ${ }^{17,18}$ Interestingly however, we found in our experimental data that these particle-like trends are not always followed for irregularly shaped surfaces. For instance, different solitary protrusions of nearly identical size and shape would in some cases have the same plasmon frequency, but in other cases not. To get insight into this phenomenon, the dominant, longitudinal plasmon modes were simulated for a series of elongated gold particles of different shapes, shown in Fig. 3b.

In agreement with earlier work, ${ }^{26}$ the black graph in Fig. $3 \mathrm{~b}$ shows a strong redshift for the plasmon resonance frequencies of gold cylinders with increasing aspect ratios. For bumps or protrusions attached to smaller or equally-sized gold volumes, the plasmon frequency follows the same trend, and depends on the total length of the particle. However, when the volume to which a protrusion is attached is large in comparison with the protrusion size, the longitudinal plasmon frequency is not a function of the total length anymore, but only depends on the size of the protrusion itself. The blue, red, and green lines in Fig. $3 b$ show this-yet unreported-effect for three differently-sized protrusions. If a protrusion is 
attached to a large volume, only the electrons closest to the protrusion are involved in the plasmon resonance, and the resonance frequency is independent of the size of the volume to which the protrusion is attached. Fig. 3c gives more details on this effect, which results from the limited skin depth of surface plasmons at these frequencies. It can be seen that even a slight elongation of a surface feature gives strongly enhanced local charge densities due to the excitation of the longitudinal surface plasmon mode. Incoming light might excite this dipole mode when components of the electric field vector point in the direction of the feature's axis of symmetry. The random orientation of protrusions in nanoporous gold and silver therefore assures strong coupling and omnidirectional light absorption at dipolar plasmon frequencies.

All the above-mentioned size and shape effects are able to modify the optical properties both in the far-field and in the near field. Not always do these two domains show similar spectral responses. As mentioned above, dark modes can be an important source of discrepancies between EELS and optical absorption spectra, which is also the case for local variations in plasmon damping. ${ }^{27}$ For our mesoporous gold, a comparison between far-field optical and near-field EELS measurements is made in Figure 4. Due to its more local nature, it should be mentioned that the EELS signal was acquired from a much smaller total area than the optical measurements, making it more susceptible to local variations. The optical spectra were calculated from Drude-Lorentz parameters that were derived from fits to optical measurements of two mesoporous gold films with morphologies and thicknesses closest to those used for the EELS measurements. ${ }^{2}$ The two optical spectra show similar overall trends, but there is certainly no perfect overlap with the EELS data. We ascribe the higher EELS intensity at low energies to damping-induced red shifts, ${ }^{27}$ and higher near-field absorption due to the excitation of dark modes with EELS.

EELS and Discrete Dipole simulations on mesoporous gold. Despite the somewhat different morphology of mesoporous gold, it shows similar plasmonic behavior in Fig. 5 as was the case for nanoporous silver. Firstly, several plasmon modes can again be observed, all linked to specific morphological features with deep sub-wavelength dimensions. Also, the resonance frequencies are often related to the size of the local volume, with low-frequency modes delocalized over large areas and highfrequency modes more confined in space. Dark modes are again present at elongated structures. From a $90 \mathrm{~nm}$ long trajectory, several major and minor plasmon modes are seen in the spectra of Fig. 5c. The plasmon mode around $1 \mathrm{eV}$, coded in pink color, is caused by a resonance in the large, irregular structure to the left of the square in Fig. 5b. This same square region was also used for simulations, to relate the EELS results with plasmon modes that would be excited by plane-wave light illumination. Using the discrete dipole scattering (DDSCAT) approach, ${ }^{28}$ the simulated optical extinction spectrum is shown in Fig. 6b, together with a sum of all EELS spectra in this region. Encouragingly, the spectra are 
in agreement on the energy of the plasmon resonances. However, the intensity of the peaks differs between experiment and simulation. A comparison of the plasmon maps at 1.45 and $2.05 \mathrm{eV}$ in Fig. 6c and Fig. 6d respectively, shows the source of this quantitative discrepancy. The computationally intensive DDSCAT simulations had to be limited to 1 million dipoles, spaced by $0.5 \mathrm{~nm}$ or less, restricting the size of the gold volume that could be used in the simulations. Fig. 6a shows the geometry of the used DDSCAT model; it can be seen that the boundaries of the model had to be set somewhat differently in comparison with the experimental situation. For example, the large volume to the left that is responsible for the strong $1 \mathrm{eV}$ resonance could only partly be included in the model, probably explaining why this resonance is much stronger in the EELS spectrum. A larger-scale calculation would correctly simulate the right response, but requires computational resources beyond our reach. On the other hand, the three-dimensionality that is lost in the EELS maps, is conserved in the DDSCAT simulations, giving a more comprehensive view of the local field enhancements.

Figure $6 \mathrm{c}$ shows that together with the central part of the structure, the lower right part of the model displays a strong plasmon resonance at $1.45 \mathrm{eV}$. This resonance is not present in the EELS map because in the experiment, the lower right section is attached to a large volume. In comparison with the simulated spectrum, therefore, the EELS spectrum at $1.45 \mathrm{eV}$ is much lower in intensity. For the more localized plasmon resonances at $2.05 \mathrm{eV}$ on the other hand, Fig. $6 \mathrm{~d}$ shows good agreement between the experiment and the simulations. A subtle difference is the enhanced field intensity in the middle of two thin, elongated features. These resonances are present in the EELS maps but not in the plane-wave simulations, indicating that they are dark modes, similar to the silver bridge in Fig. 1. The qualitative agreement between EELS experiment and plane-wave simulations illustrates that at even at deepsubwavelength dimensions, plane-wave, far-field light illumination and EELS can excite the same localized plasmon modes, ${ }^{29}$ except of course for dark modes which are not directly accessible with farfield techniques. The nanoporous silver and gold studied here generally do not consist of filaments, but of metal aggregates with protrusions that couple relatively easily with light and where dark modes are a minority in the dominant surface plasmon resonances.

\section{CONCLUSIONS}

It was demonstrated that the anomalous optical characteristics of nanoporous silver and gold find their origin in the excitation of localized surface plasmon resonances. At deep sub-wavelength spatial dimensions, the excitation of localized plasmon resonances causes relatively narrow-band absorption, where the plasmon resonance energy varies strongly between different locations. This nanoscale polychromaticity was visualized with experimental EELS mapping and DDSCAT simulations. The broadband absorption of these materials in the far field was demonstrated to be the aggregate of many 
local, narrow-band absorption events. Size and shape of the surface protrusions are parameters for tuning the absorption color, similar to the optical activity of individual gold and silver particles. In addition to this effect, it was shown that the volume to which the protrusions are attached is also important as a tuning parameter. As a result, direct control over the broadband absorption characteristics of these materials can be obtained by tuning the growth parameters, which were shown earlier to control the morphology of mesoporous gold and silver. ${ }^{2}$ The findings in this work allow the controlled design of thin films and coatings that have strong broadband absorption, while-for example-remaining reflective in custom-designed wavelength bands. Direct applications of these findings can be foreseen in photovoltaics, ${ }^{30}$ where the inclusion of a well-tuned mesoporous film in (or adjacent to) the photoactive semiconductor will optimize the power conversion efficiency. Finally, mesoporous gold is biocompatible; its absorption characteristics can be tuned and optimized for various biosensing applications, ${ }^{31}$ without the need for complicated assembly procedures.

\section{METHODS}

Metal synthesis. Mesoporous gold specimens were prepared by depositing $\mathrm{AuAl}_{2}$ on cleaved rock salt, using ionassisted co-deposition of pure gold and aluminum with high vacuum dc magnetron sputtering. The base pressure was better than $\sim 10^{-6}$ Torr, with $2 \mathrm{mTorr}$ argon as inlet gas, a rotating substrate at $400^{\circ} \mathrm{C}$ and radio frequency bias to modulate the final morphology. Aluminum was de-alloyed from $\mathrm{AuAl}_{2}$ by immersing the films in $\mathrm{NaOH}(0.2 \mathrm{M})$ solution, leaving percolating pores in the gold matrix. ${ }^{32}$ The thin, mesoporous gold film was then floated off onto a copper cross grating TEM support grid for EELS analysis. Thin, nanoporous silver film was directly deposited on cleaved rock salt using de magnetron sputtering, but floated off onto a TEM specimen grid of ultrathin carbon (Ted Pella); the fragility of the porous silver film required more mechanical support, which the carbon provided. The ultrathin carbon gave a low background signal in the EELS spectrum, comprising a few percent of the spectral intensity in areas with plasmonic activity. Some damping of the plasmon amplitude is likely to occur as a result of the carbon substrate. However, experimental data sets where part of the carbon film was absent in some locations (not shown here) did not display any observable amplitude modulations or shifts in plasmon energy for the same modes, validating the minor influence of the substrate on the results.

Electron energy-loss spectroscopy. EELS measurements were done using the Binned Gain Averaging method described in detail elsewhere, ${ }^{24}$ using a $100 \mathrm{kV} \mathrm{VG} \mathrm{STEM} \mathrm{-} \mathrm{Gatan} \mathrm{Enfina} \mathrm{system} \mathrm{with} 5$ ms acquisition time per spectrum, or a total of around 9 minutes for the large gold and silver maps. The cold field emission gun was operated with an electron extraction voltage $20 \%$ below its saturation limit. In this way, an electron beam was obtained with a monochromaticity of $0.25 \mathrm{eV}$ or better, measured as width of the zero-loss peak at half its maximum intensity. The STEM probe diameter used was measured to be 2-3 nm, and STEM convergence and EELS collection semi-angles of respectively 11 and 3 mrad were used for the experiments. The strong background signal in the EELS spectrum from the intensity tail of the zero-loss peak was removed by fitting a pre-measured zero-loss peak to each spectrum between 0.65 and $0.85 \mathrm{eV}$, and subtracting its intensity. It is worth noting that due to the high zero-loss peak signal at lower energies, the variance in the extracted plasmon amplitude increases towards low energies. The shown spectra were obtained by summing 90 to 150 single spectra and normalizing (i.e. dividing by 90 to 150 ) the spectra in each line trace to the same total acquisition time. Plasmon maps were obtained by 
integrating the EELS intensity in each pixel from a $0.15 \mathrm{eV}$ wide energy window. Intensity maps were plotted in linear scale, and each map was color-coded as indicated in the figures.

Simulations. For the non-retarded simulations, analytical expressions for the energy-loss probability were derived from

Illman et al. $^{33}$ Simulations that include retardation effects were done using the boundary element method, specialized to systems of rotational symmetry. ${ }^{34}$ In this method, the surface is divided into thin cylindrical rings centered about the axis of symmetry and fields are written as Fourier series in the azimuthal angle. It was found that good accuracy could be obtained if the rings are of height $1 \mathrm{~nm}$ and the zeroth- and first-orders of the Fourier series were included. The virtual STEM electron probe is located $1 \mathrm{~nm}$ to the right of each structure, exciting a longitudinal plasmon oscillation in the horizontal plane.

DDSCAT simulations were done by first digitizing a STEM HAADF image of the film, to build a 3D geometric model. Minimum HAADF intensity was set to correspond to zero thickness and maximum HAADF intensity to $23 \mathrm{~nm}$ thick gold. This 3D model was used as a target geometry in the DDSCAT program, the discrete dipole approximation (DDA) code of Draine and Flatau, ${ }^{28,35}$ which has the particular advantage that it can be applied to arbitrarily-shaped targets. In the DDA approach, the nanostructure is approximated by a three-dimensional array of dipoles and the response of this array of dipoles to an incident field is then determined numerically. The DDSCAT program has been found to give accurate results, provided that a sufficiently small spacing for the dipoles is used. For the complex geometry used here, a $0.5 \mathrm{~nm}$ mesh was used for the 3D model, necessarily limiting the total volume that can be analyzed. The resulting electric fields were extracted using the DDFIELD utility of Draine and Flatau, and then visualized using our own software.

Acknowledgements. Discussions with Wilfried Sigle (MPI, Stuttgart) and Joel Yang (IMRE, Singapore) are kindly acknowledged; Abbas Maaroof (UT, Sydney) is thanked for help in specimen fabrication. We thank Paul Thomas (Gatan, UK) for EELS-processing scripts.

\section{REFERENCES}

1. Raether, H. Surface Plasmons on Smooth and Rough Surfaces and on Gratings; Springer, Berlin, 1988.

2. Maaroof, A. I.; Gentle, A.; Smith, G. B.; Cortie, M. B. Bulk and surface plasmons in highly nanoporous gold films. J. Phys. D: Appl. Phys. 2007, 40, 5675-5682.

3. Teperik, T. V.; García de Abajo, F. J.; Borisov, A.G.; Abdelsalam, M.; Bartlett, P. N.; Sugawara, Y.; Baumberg, J. J. Omnidirectional absorption in nanostructured metal surfaces. Nat. Photonics 2008, 2, 299-301.

4. Maier, S. A.; Brongersma, M. L.; Kik, P. G.; Meltzer, S.; Requicha, A. A. G.; Atwater, H. A. Plasmonics - a route to nanoscale optical devices. Adv. Mater. 2001, 13, 1501-1505.

5. García de Abajo, F. J. Colloquium: Light scattering by particle and hole arrays. Rev. Mod. Phys. 2007, 79, 1267-1290.

6. Schuller, J. A.; Barnard, E. S.; Cai, W.; Jun, Y. C.; White, J. S.; Brongersma, M. L. Plasmonics for extreme light concentration and manipulation. Nat. Mater. 2010, 9, 193-204. 
7. Genet, C; Ebbesen, T. W. Light in tiny holes. Nature 2007, 445, 39-46.

8. Sönnichsen, C.; Franzl, T.; Wilk, T.; von Plessen, G.; Feldmann, J. Drastic reduction of plasmon damping in gold nanorods. Phys. Rev. Let. 2002, 88, 077402.

9. Klar, T.; Perner, M.; Grosse, S.; von Plessen, G.; Spirkl, W.; Feldmann, J. Surface-plasmon resonances in single metallic nanoparticles. Phys. Rev. Lett. 1998, 80, 4249-4252.

10. Stiegler, J. M.; Abate, Y.; Cvitkovic, A.; Romanyuk, Y. E.; Huber, A. J.; Leone, S. R.; Hillenbrand R. Nanoscale infrared absorption spectroscopy of individual nanoparticles enbled by scattering-type near-field microscopy. ACS Nano 2011, 5, 6494-6499.

11. Ruthermann, G. Diskrete energieverluste mittelschneller Elektronen beim durchgang durch dünne Folien. Ann. Phys. 1948, 437, 113-134.

12. Pines, D.; Bohm, D. A collective description of electron interactions: II. Collective vs. individual particle aspects of the interactions. Phys. Rev. 1952, 85, 338-353.

13. Ritchie, R. H. Plasma losses by fast electrons in thin films. Phys. Rev. 1957, 106, 874-881.

14. Batson, P. E. Surface plasmon coupling in clusters of small spheres. Phys. Rev. Lett. 1982, 49, 936-940.

15. Wang, Z. L.; Cowley, J. M. Generation of surface plasmon excitation of supported metal particles by an external electron beam. Ultramicrosopy 1987, 21, 347-366.

16. Nelayah, J.; Kociak, M.; Stéphan, O.; García de Abajo, F. J.; Tencé, M.; Henrard, L.; Taverna, D.; Pastoriza-Santos, I.; Liz-Marzán, L. M.; Colliex, C. Mapping surface plasmons on a single metallic nanoparticle. Nat. Phys. 2007, 3, 348-353.

17. Koh, A. L.; Fernández-Domínguez, A. I.; McComb, D. W.; Maier, S. A.; Yang, J. K. W. High-resolution mapping of electron-beam-excited plasmon modes in lithographically defined gold nanostructures Nano Lett. 2011, 11, $1323-1330$.

18. Bosman, M.; Keast, V. J.; Watanabe, M.; Maaroof, A. I.; Cortie, M. B. Mapping surface plasmons at the nanometre scale with an electron beam. Nanotechnology 2007, 18, 165505.

19. Rossouw, D.; Couillard, M.; Vickery, J. Kumacheva, E.; Botton, G.A. Multipolar plasmonic resonances in silver nanowire antennas imaged with a subnanometer electron probe. Nano Lett. 2011, 11, 1499-1504.

20. Stockman, M. I.; Faleev, S. V.; Bergman, D. J. Localization versus delocalization of surface plasmons in nanosystems: can one state have both characteristics? Phys. Rev. Lett. 2001, 87, 167401.

21. Chu, M. W.; Myroshnychenko, V.; Chen, H. C.; Deng, J.-P.; Mou, C.-Y.; García de Abajo, F. J. Probing bright and dark surface-plasmon modes in individual and coupled noble metal nanoparticles using an electron beam. Nano Lett. 2009, 9, 399404.

22. Liu, N.; Langguth, L.; Weiss, T.; Kästel, J.; Fleischhauer, M.; Pfau, T.; Giessen, H. Plasmonic analogue of electromagnetically induced transparency at the Drude damping limit. Nat. Mater. 2009, 8, 758-762.

23. García de Abajo, F. J. Optical excitations in electron microscopy. Rev. Mod. Phys. 2010, 82, 209-275. 
24. Bosman, M.; Keast, V. J. Optimizing EELS acquisition. Ultramicroscopy 2008, 108, 837-846.

25. Sigle, W.; Nelayah, J.; Koch, C. T.; van Aken, P.A. Electron energy losses in Ag nanoholes-from localized surface plasmon resonances to rings of fire. Optics Lett. 2009, 34, 2150-2152.

26. Myroshnychenko, V.; Rodríguez-Fernández, J.; Pastoriza-Santos, I.; Funston, A. M.; Novo, C.; Mulvaney, P.; LizMarzán, L.; García de Abajo, F. J. Modelling the optical response of gold nanoparticles. Chem. Soc. Rev. 2008, 37, 17921805.

27. Zuloaga, J.; Nordlander, P. On the energy shift between near-field and far-field peak intensities in localized plasmon systems. Nano Lett. 2011, 11, 1280-1283.

28. Draine, B. T.; Flatau, P. J. Discrete-dipole approximation for scattering calculations. J. Opt. Soc. Am. A 1994, 11, 14911499.

29. García de Abajo, F. J.; Kociak, M. Probing the photonic local density of states with electron energy loss spectroscopy. Phys. Rev. Lett. 2008, 100, 106804.

30. Atwater, H. A.; Polman, A. Plasmonics for improved photovoltaic devices. Nat. Mater. 2010, 9, $205-213$.

31. Lang, X.; Qian, L.; Guan, P.; Zi, J.; Chen, M. Localized surface plasmon resonances of nanoporous gold. Appl. Phys. Lett. 2011, 98, 093701.

32. Supansomboon, S.; Maaroof, A. I.; Cortie, M. B. "Purple glory": The optical properties and technology of $\mathrm{AuAl}_{2}$ coatings. Gold Bulletin 2008, 41, 296-304.

33. Illman, B. L.; Anderson, V. E.; Warmack, R. J.; Ferrell, T. L. Spectrum of surface-mode contributions to the differential energy-loss probability for electrons passing by a spheroid. Phys. Rev. B 1988 38, 3045-3049.

34. García de Abajo, F. J.; Howie, A. Retarded field calculation of electron energy loss in inhomogeneous dielectrics. Phys. Rev. B 2002, 65, 115418

35. Draine, B. T.; Flatau, P. J. User Guide for the Discrete Dipole Approximation Code DDSCAT 6.1. URL: http://arxiv.org/abs/astro-ph/0409262 2004. 
Figure 1 (single column)
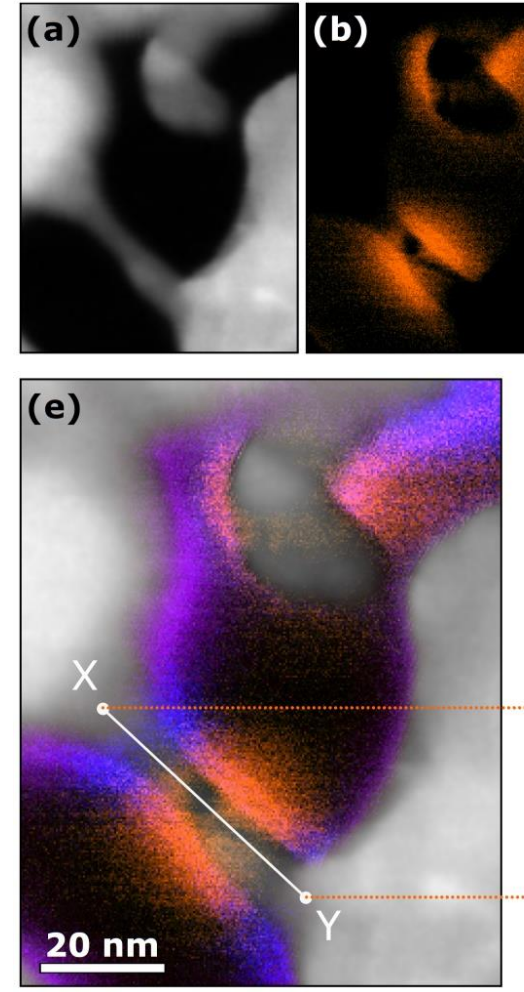

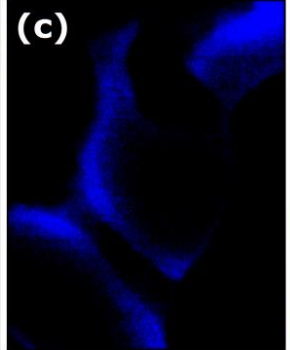

\section{(d)}

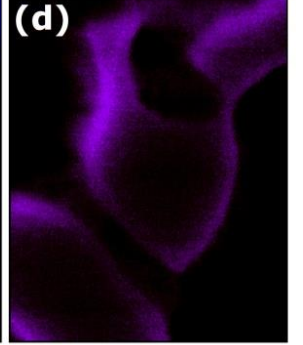

(f)

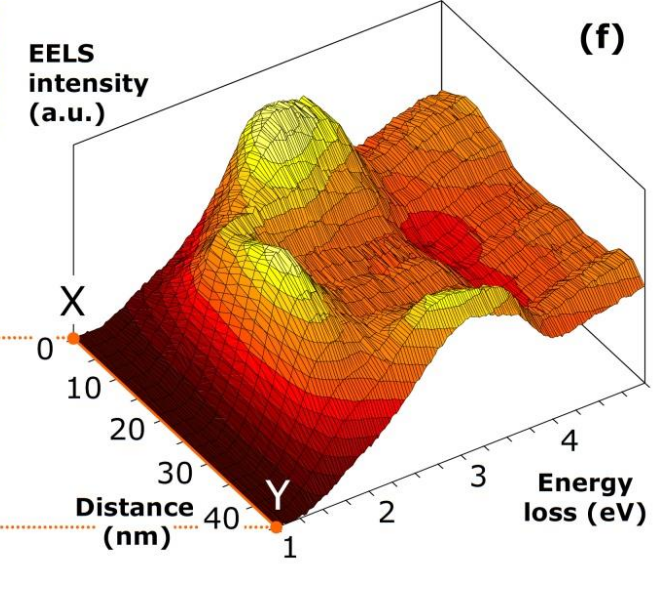

Figure 1. Spectroscopy and mapping of local surface plasmon modes in porous silver. (a) HAADF STEM image, showing the silver and (in black) the empty pores. (b-d) Plasmon maps at 1.90, 2.90, and $3.45 \mathrm{eV}$ respectively, obtained from $0.15 \mathrm{eV}$ EELS intensity windows at these energies. The EELS intensity is coded according to the color of light that would be absorbed by this plasmon under dipolar mode resonance; the near-UV absorption is indicated in violet. (e) Overlay of figures a-d. (f) A series of EELS spectra showing the plasmon modes at 1.90 and $2.90 \mathrm{eV}$, taken along the length of the $45 \mathrm{~nm}$ long and 7-10 nm wide, silver bridge. 


\section{Figure 2 (single column)}
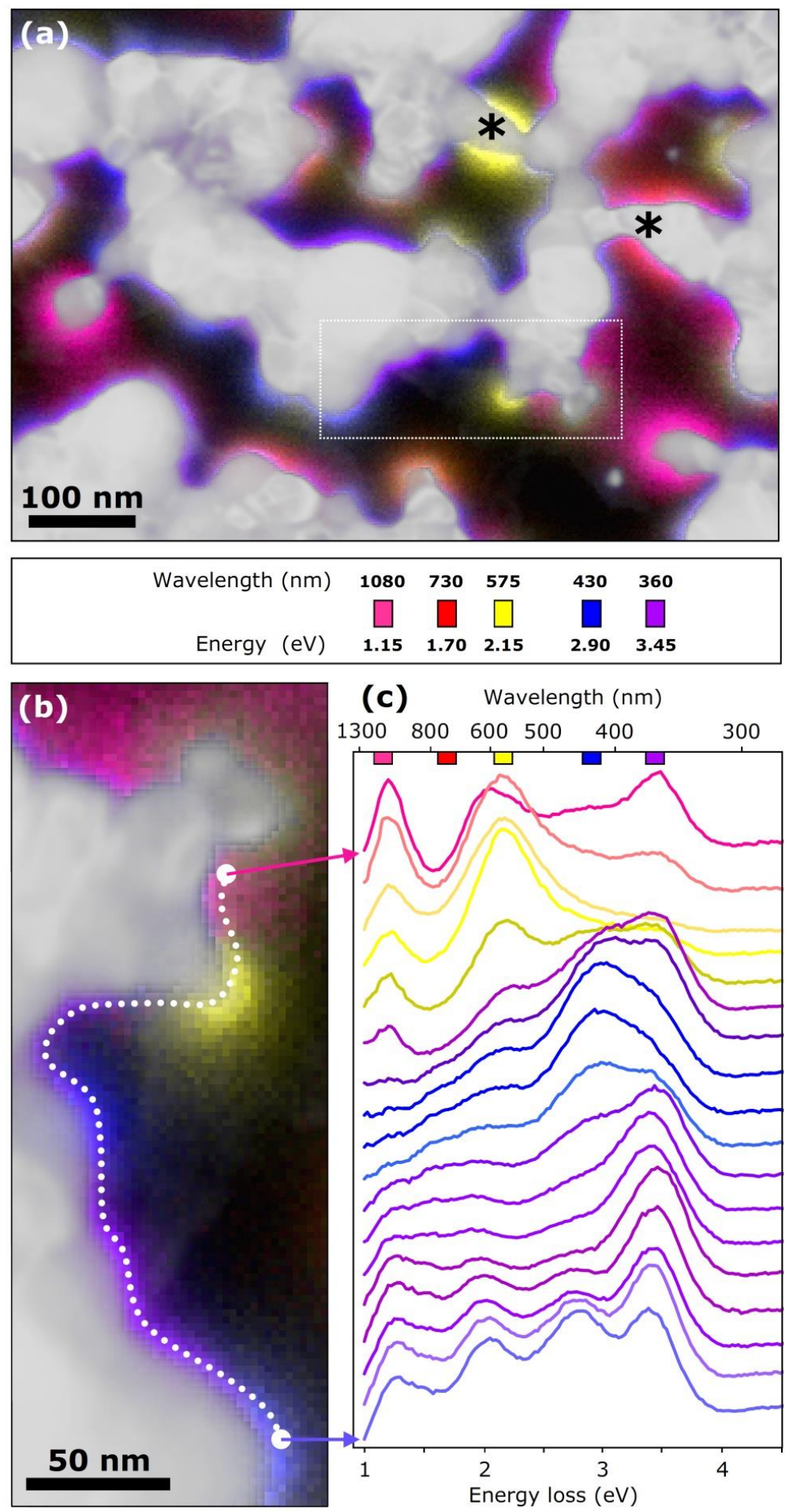

Figure 2. Local surface plasmon modes in mesoporous silver. (a) Overlay of the HAADF STEM image with five colorcoded EELS plasmon maps. The legend shows the $0.15 \mathrm{eV}$ energy windows as well as the colors used for the five EELS maps. The colors correspond to the color of the light that would locally be absorbed by dipolar plasmon resonances. Absorption in the near-infrared (wavelength $=1080 \mathrm{~nm}$ ) is indicated in pink; near-UV absorption (wavelength $=360 \mathrm{~nm}$ ) is indicated in violet. The asterisks indicate possible dark modes at elongated silver bridges. (b) Detail from figure a, with a 300 $\mathrm{nm}$ trajectory indicated by the dotted line. (c) EELS spectra from the same trajectory. Colors of the spectra correspond with the locations on the map. 

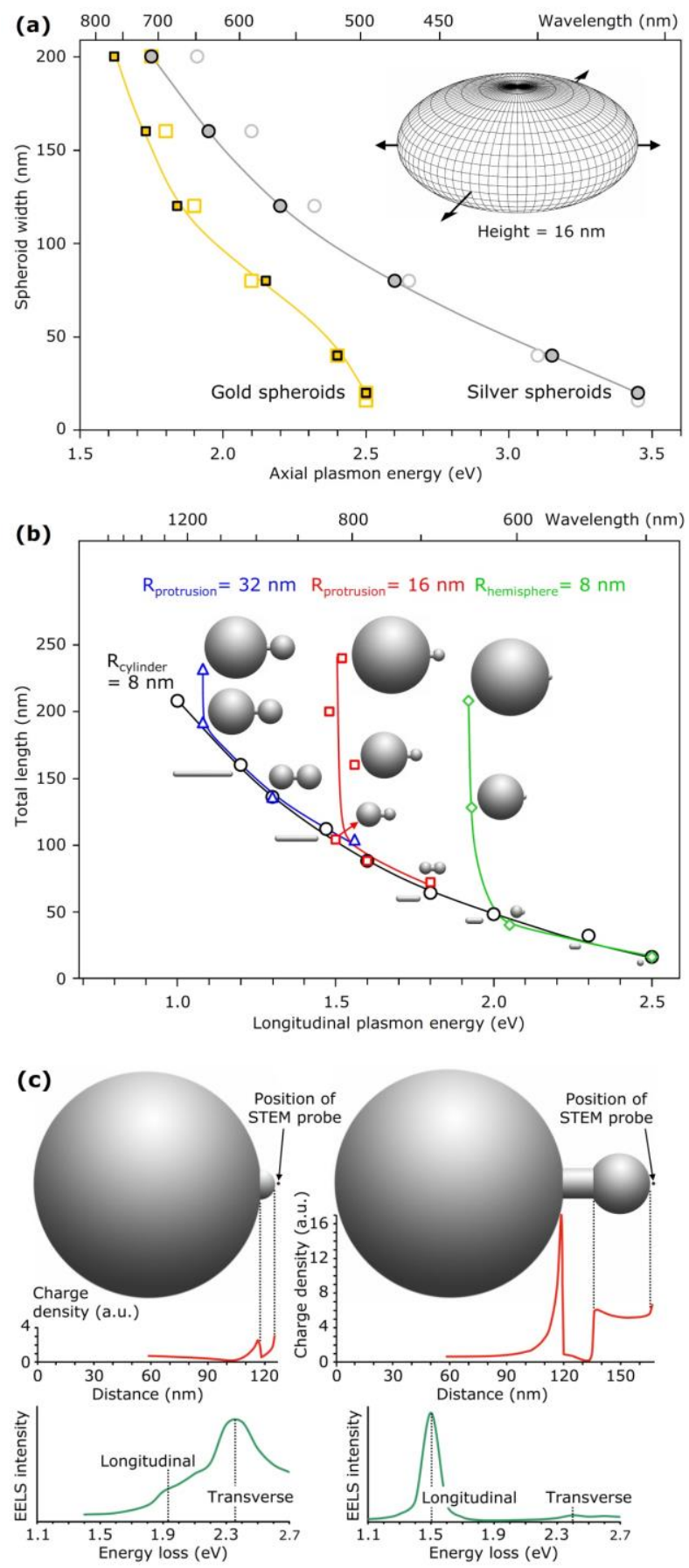

Figure 3. Simulated axial and longitudinal plasmon modes for various gold and silver geometries. (a) Axial plasmon energy as a function of width for $16 \mathrm{~nm}$ high gold and silver oblate spheroids. Open symbols are from simulations that ignore retardation; filled symbols include retardation effects. (b) Longitudinal plasmon energy as a function of total length for various gold geometries; simulations include retardation effects. Black: cylinders with radius of $8 \mathrm{nn}$ and various lengths; Green: a hemisphere with radius $8 \mathrm{~nm}$ protruding from different volumes; Red: a sphere with radius $16 \mathrm{~nm}$ and a cylinder with radius and length $8 \mathrm{~nm}$ protrude from different volumes; Blue: same, but with a $32 \mathrm{~nm}$ sphere. The size of the different geometries drawn in grey next to the data points is scaled to be mutually comparable. (c) Simulated charge distribution (red plot) and EELS spectrum (green) for a small protrusion (left, hemisphere with radius $8 \mathrm{~nm}$ ) and for a large protrusion (right, sphere with radius $16 \mathrm{~nm}$ plus $8 \mathrm{~nm}$ cylinder) protruding from a sphere with radius $60 \mathrm{~nm}$. 
Figure 4 (single column)

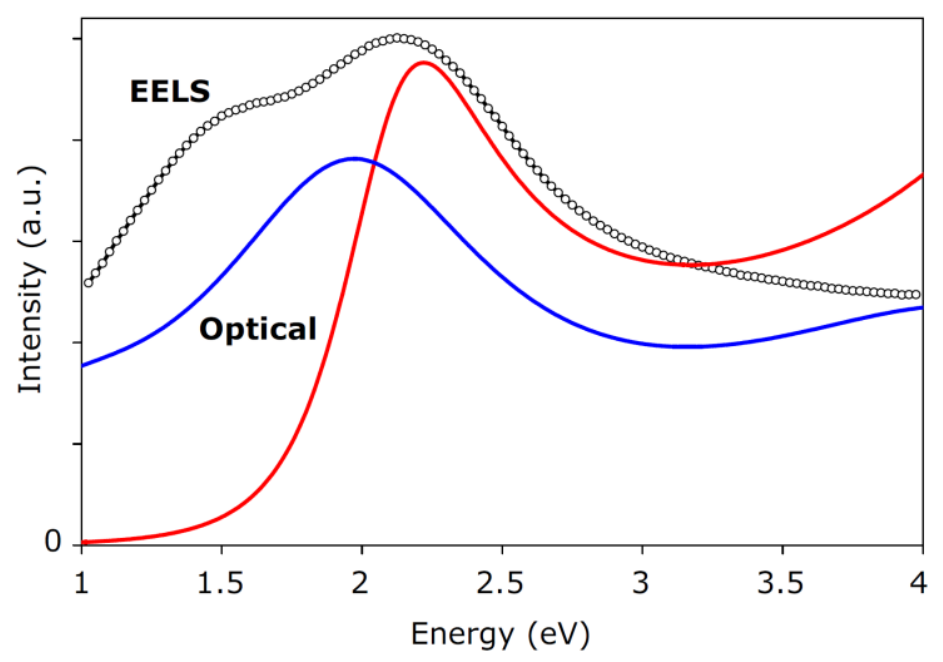

Figure 4. Comparing EELS and optical data from mesoporous gold. The black open circles show the summation of EELS spectra from a $386 \times 386 \mathrm{~nm}^{2}$ area, compared to the expected low-loss spectrum, derived from optical measurements. ${ }^{2}$ Red plot: mesoporous gold grown with ion-assistance and a maximum thickness of about $34 \mathrm{~nm}$. Blue plot: mesoporous gold grown without ion assistance and a maximum thickness of $37.5 \mathrm{~nm}$. 

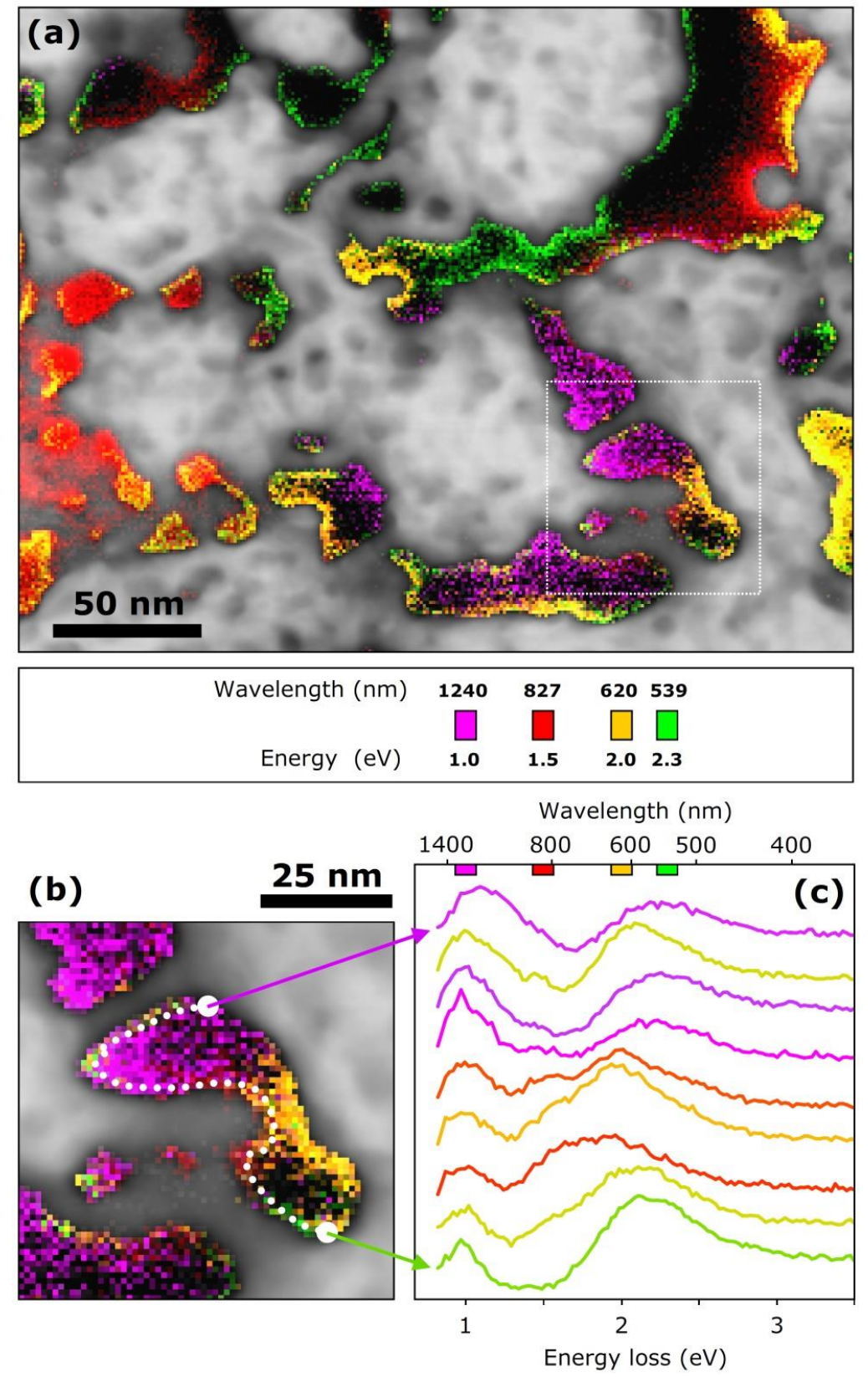

Figure 5. Localized surface plasmon resonances in mesoporous gold. (a) Overlay of the HAADF STEM image and EELS plasmon maps from the four dominant absorption bands. The legend indicates the $0.15 \mathrm{eV}$ energy windows for the EELS maps and is color-coded according to the color that would be absorbed if the plasmons were excited by white light, with the near-infrared $1 \mathrm{eV}$ plasmon mode coded in pink color. (b) Detail from Figure a, with a $90 \mathrm{~nm}$ trajectory indicated by the dotted line. (c) EELS spectra from the trajectory indicated in b. Colors correspond to the locations on the trajectory. 
Figure 6 (double column)

(a)

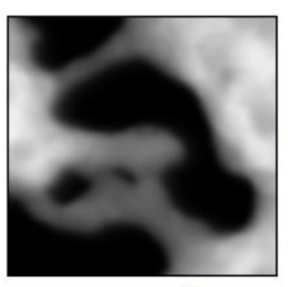

HAADF STEM $25 \mathbf{~ n m}$

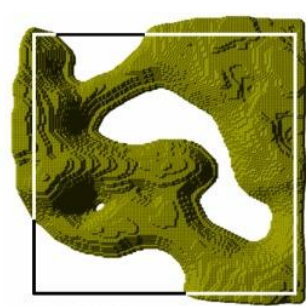

DDSCAT model
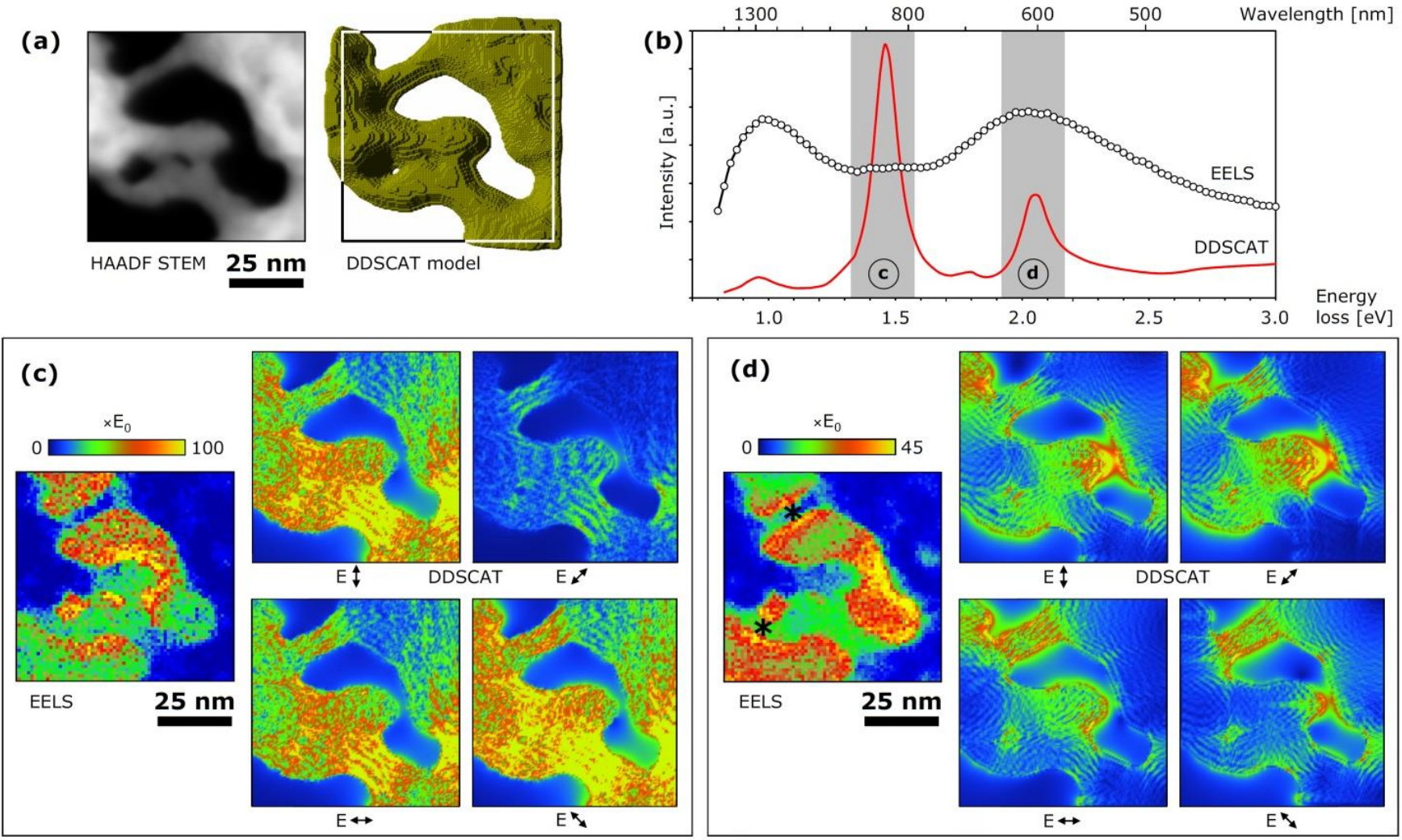

(d)
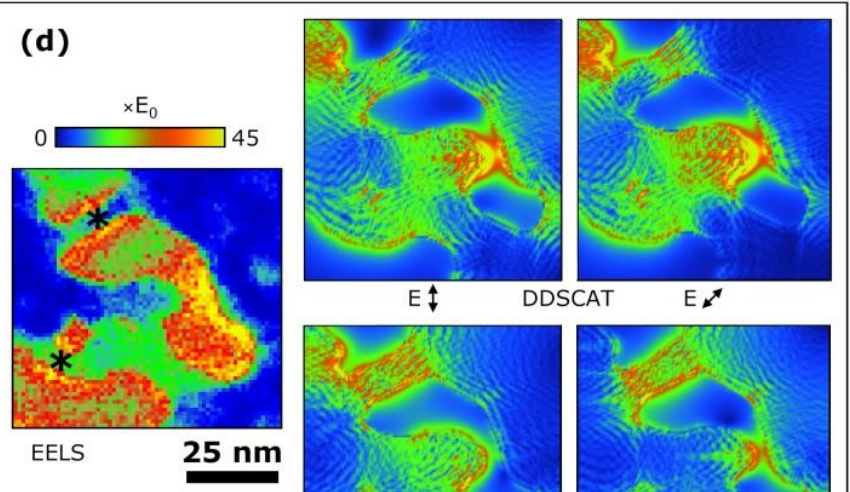

E

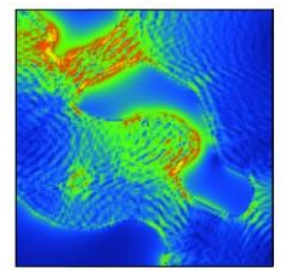

$E \leftrightarrow$

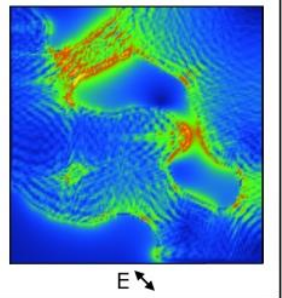

Figure 6. Plasmon modes excited by an electron probe and by plane-wave light illumination. (a) HAADF STEM image, and gold dipole 3D model. The whole volume used for the simulations is shown; the square box indicates the displayed areas in the DDSCAT maps. (b) In red: DDSCAT calculated optical extinction spectrum (in this example, using a plane wave illumination at orientation $0^{\circ}$ from the vertical), compared with a sum of all EELS spectra in the square studied area. Grey shading: energy windows that were used for the maps in Figs. c and d. (c, left) EELS intensity map at $1.45 \mathrm{eV}$. (c, right) DDSCAT absorption maps at wavelength $850 \mathrm{~nm}$, using four illumination angles $\left(0^{\circ}, 45^{\circ}, 90^{\circ}\right.$ and $135^{\circ}$ from the vertical), as indicated by the arrows. (d, left) EELS intensity map at $2.05 \mathrm{eV}$. (d, right) DDSCAT absorption maps at wavelength $605 \mathrm{~nm}$. The color table used for the maps shows the calculated electric field strength compared to the illuminating field strength; the same color table was used for the EELS maps. The asterisks indicate possible dark modes at elongated gold bridges. 
TOC graphic

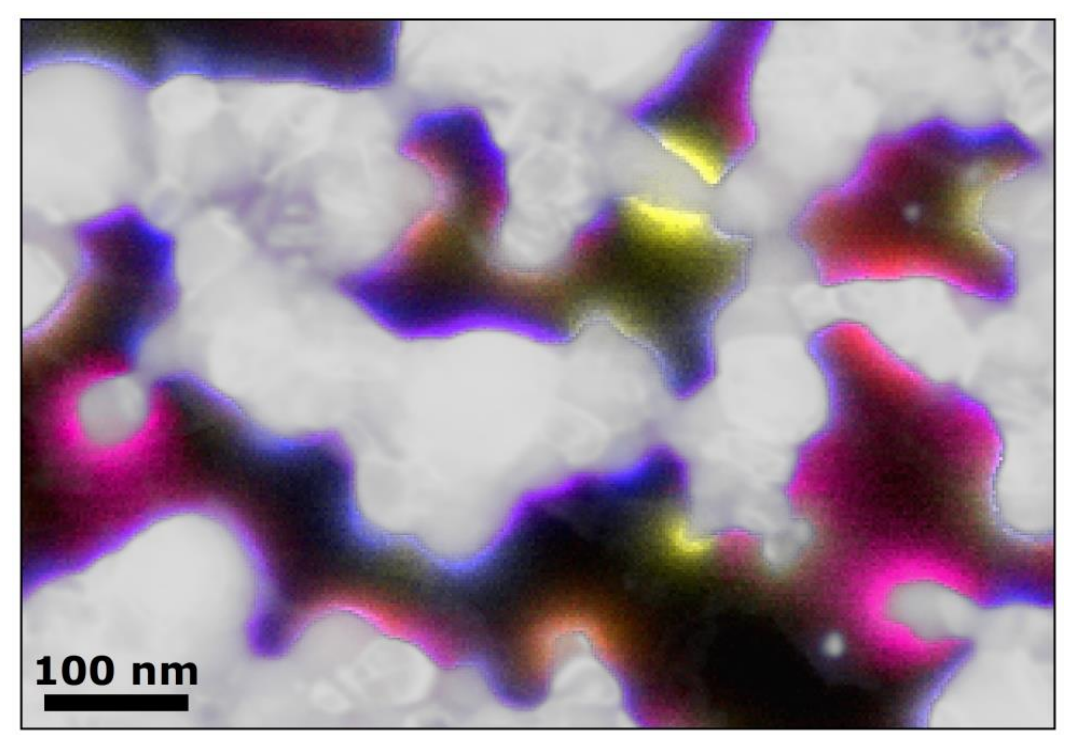

